\title{
High pressure Hugoniot measurements using converging shocks
}

\author{
J. L. Brown, ${ }^{1, a)}$ G. Ravichandran, ${ }^{1}$ W. D. Reinhart, ${ }^{2}$ and W. M. Trott ${ }^{2}$ \\ ${ }^{1}$ California Institute of Technology, Pasadena, California, 91125, USA \\ ${ }^{2}$ Sandia National Laboratories, Albuquerque, New Mexico 87185, USA
}

(Received 22 February 2011; accepted 11 April 2011; published online 12 May 2011)

\begin{abstract}
Plate impact experiments are a powerful tool in equation of state development, but are inherently limited by the range of impact velocities accessible to the facility. In an effort to dramatically increase the range of pressures which can be studied with available impact velocities, a new experimental technique is examined. The target plate is replaced by a composite assembly consisting of two concentric cylinders. The target is designed such that the initial shock velocity in a well-characterized outer cylinder is higher than in the inner cylinder material of interest. Conically converging shocks will be generated at the interface due to the impedance mismatch between the two materials and axisymmetric geometry. Upon convergence, an irregular reflection occurs and the conical analog of a Mach reflection develops. This Mach reflection grows until it reaches a steady state, at which point the high pressure state in the Mach disk can be measured using velocity interferometry and impedance matching techniques. The technique is demonstrated by studying the shock response of copper. A strong confinement setup utilizes a low impedance 6061-T6 aluminum outer cylinder and the weak confinement case features a higher impedance molybdenum outer cylinder. The experimental results for copper are in good agreement with a simple analytical model, numerical simulations, and data in the literature. The possibility of utilizing full field measurements to make multiple Hugoniot measurements is also examined. (C) 2011 American Institute of Physics. [doi:10.1063/1.3590140]
\end{abstract}

\section{INTRODUCTION}

It has long been recognized that high pressure equation of state (EOS) measurements can be determined by subjecting the material to a one-dimensional plane shock wave. ${ }^{1}$ The resulting equilibrium state behind the shock wave can be characterized by making two experimental measurements of the jump in material properties, typically shock speed and particle velocity, and relating the rest of the thermodynamic properties with the Rankine-Hugoniot conservation equations. Shock waves of this type are usually generated by the planar impact of flat plates. Because the resulting pressure of the shock wave generated in these types of experiments is determined by the impact velocity, there has been considerable interest in finding techniques which can be used to extend the range of the impact velocities. Gun systems, for example, were traditionally limited by two-stage launcher technology to velocities of approximately $7 \mathrm{~km} / \mathrm{s}^{2}$, until the development of the hypervelocity launcher ${ }^{3}$ which utilizes a graded density impactor to produce quasi-isentropic loading of a third stage flyer to velocities of $12 \mathrm{~km} / \mathrm{s}$. Later, isentropic loading was magnetically generated using the Sandia National Laboratories $\mathrm{Z}$ accelerator ${ }^{4}$ where flyer velocities of over $25 \mathrm{~km} / \mathrm{s}$ have been obtained. ${ }^{5}$

Significant pressure increases can also be achieved through the interaction of shock waves. The nonlinear nature of shock waves can result in irregular reflections under special conditions, a phenomenon first discovered by Ernst Mach in 1869. The subject is thoroughly reviewed in gases

\footnotetext{
a) Author to whom correspondence should be addressed. Electronic mail: jlbrown@caltech.edu.
}

by Bleakney and Taub, ${ }^{6}$ and the first experimental observations of Mach reflections in metals were made by Al'tshuler et al. ${ }^{7}$ Later, Fowles and Isbell ${ }^{8}$ produced conically converging shocks in metal cylinders by detonating the front surface of a surrounding high explosive cylinder. The resulting Mach reflection was measured with a streak camera viewing the rear surface, for which it was estimated that there were elevated pressure states in the Mach disk. Similar measurements of the Mach configuration using the same idea were made in Plexiglas cylinders using a flash gap technique by Adadurov et al. ${ }^{9}$ This method was later extended to mechanical impact testing, ${ }^{10}$ where composite cylinders were used to generate the converging shocks and subsequent Mach reflection, creating extreme compaction in recovery experiments.

In the present study, the feasibility of using Mach reflections to make accurate high pressure EOS measurements is investigated. Oblique shock relations can be used to solve for the steady state Mach configuration which is further validated by numerical simulations. Proper design of the experiment results in an essentially planar Mach disk which travels at a speed equal to the velocity of the plane shock in the outer cylinder. As a result, the shock speed can be directly calculated from the impact configuration as long as the outer cylinder and impactor materials are well-characterized. Thus, determination of the shocked state behind the Mach disk requires only a single measurement of the particle velocity.

The experimental technique is described in Sec. II, which includes the details of the target configuration and the diagnostics. This is followed in Sec. III with a simple analytical model based on shock polars to calculate the steady state Mach configuration. Numerical simulations of the 
experiment are presented in Sec. IV and is followed by a discussion of the effect of strength on interpreting the results in Sec. V. Section VI contains the experimental results, including VISAR and ORVIS measurements, and the discussion. The conclusions for the present study are presented in Sec. VII.

\section{EXPERIMENTAL TECHNIQUE}

\section{A. Target configuration}

Experiments were performed on conventional single stage smooth bore powder guns with bore diameters of either 89 or $37 \mathrm{~mm}$. In both cases projectile velocities are limited to about $2 \mathrm{~km} / \mathrm{s}$. Flat plates were used as impactors and consisted of either 6061-T6 aluminum or 304 stainless steel. The impactors were launched into the Mach lens configuration, which utilizes the composite target schematically shown in Fig. 1. The assembly consists of an inner cylinder surrounded by a concentric outer cylinder. After impact, a plane shock is generated at the front of the target. The materials are selected such that the shock speed in the outer cylinder is higher than in the inner material of interest. The resulting impedance mismatch at the cylinder's interface produces a conical shock wave which converges on the axis of the inner cylinder. Upon convergence, the conical analog of a Mach reflection has been shown to occur. ${ }^{8-11}$ The Mach wave consists of the incident shock joining with the reflected shock and Mach stem to form the stable shock solution shown in Fig. 1. The axisymmetric geometry results in a relatively plane Mach disk at the center of the inner cylinder, for which there is a significant increase in pressure over the initial shocked state. The converging shocks and transient growth of the Mach reflection occurs over a distance approximately equal to three times the inner cylinder diameter, ${ }^{10}$ at which point the shock configuration becomes steady in time and the Mach disk reaches a limiting size and velocity.

Two assemblies have been examined in detail. The assemblies utilize an oxygen-free high-conductivity copper inner cylinder (material of interest), with either a 6061-T6 aluminum or molybdenum outer cylinder (which are assumed to be well-characterized). The target dimensions are optimized to obtain full development of the Mach reflection, while ensuring that the edge release waves from the outer cylinder do not reach the inner cylinder until well after the Mach disk has reached the rear surface, where the measurements are obtained. These dimensions were verified using the numerical simulations discussed in the next section. The
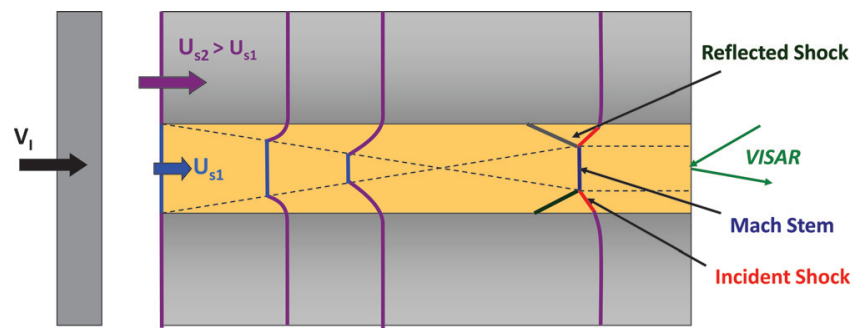

FIG. 1. (Color online) Mach lens target configuration. A plane shock is generated with a standard plate impact at the left of the target. specimens are prepared by machining the copper inner cylinder such that it is nominally $0.002^{\prime \prime}$ larger in diameter and $0.005^{\prime \prime}$ longer than the reamed hole in the outer cylinder. The inner cylinder is then coated in a layer of low viscosity epoxy and press fit into the outer cylinder, generally resulting in a small amount of copper being shaved off of the diameter and ensuring good contact between the two materials. The extra length of the inner cylinder is then sanded off and the specimens are lapped to ensure flatness.

Copper was selected as an ideal material to validate the technique because the shock properties are well known, ${ }^{12-15}$ the strength is low, ${ }^{16}$ the shock impedance is easily bracketed by other metals, and copper has been used in similar explosive configurations. ${ }^{8}$ The effect of the confining material is shown in subsequent sections, where the behavior of the lower impedance aluminum confinement is very different from that of the higher impedance molybdenum.

\section{B. Diagnostics}

Quantities measured during the experiment include the projectile velocity, impact planarity, and free surface velocity. Projectile velocity is measured either by a light interruption system or staggered electric shorting pins and is estimated to be accurate to within 1\%. Similar errors are obtained when four equally spaced shorting pins are used along the outer diameter of the impact surface of the target to calculate the impactor tilt. ${ }^{17}$ The planarity in these experiments was generally less than 3 milliradians.

Velocity interferometry was used in either single point or full-field measurements using the VISAR ${ }^{18}$ or ORVIS ${ }^{19}$ techniques, respectively. The precision in measuring the particle velocity is consistently reported as being less than $2 \%$ for the VISAR. ${ }^{20}$ To fully verify the single point measurements, some experiments utilized a double delay system, where collected light is sent into two interferometers with different sensitivities. Analysis of this data results in no ambiguity in the fringe jumps, so a distinct solution of the free surface velocity is obtained without any assumptions. The precision of the ORVIS depends not only on the fringe sensitivity, but also on the setup of the streak camera. In these experiments, an $8 \mathrm{~mm}$ line was imaged with a $5 \mu$ s sweep. The streak camera's CCD collects 1344 pixels in the spatial direction and 1024 pixels over the temporal sweep. Thus, the estimated resolution of this system is approximately $24 \mu \mathrm{m}$ and $5 \mathrm{~ns}$ in space and time, respectively.

\section{SHOCK POLAR ANALYSIS}

Assuming a completely hydrodynamic response for both the inner and outer cylinders, a calculation of the steady state wave configuration is possible through the use of oblique shock relations. Using a classic treatment of oblique shocks, ${ }^{21}$ the shock is viewed in the stationary frame shown in Fig. 2(b). The upstream flow velocity, $q_{0}$, is deflected an angle $\theta$ by the oblique shock with angle $\beta$ relative $q_{0}$. The downstream flow conditions are determined from the fact that the normal components of the velocities must follow the normal shock jump conditions, while the tangential components are conserved. For solids, a convenient form of the 


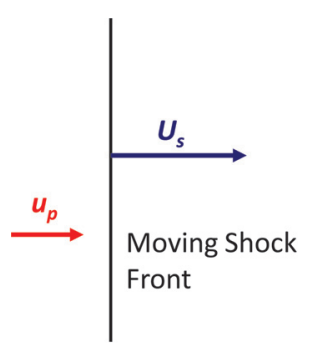

(a)

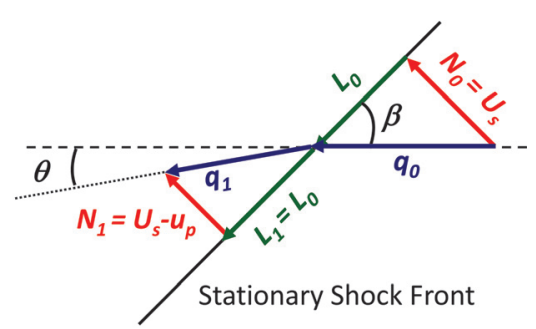

(b)
FIG. 2. (Color online) Shock configurations for (a) a normal shock in the Lagrangian frame and (b) an oblique shock in the Eulerian frame.

oblique shock relations is given by relating the normal shock jump conditions commonly reported by the shock compression community,

$$
\begin{gathered}
\tan \theta=\frac{u_{p}\left(q_{0}^{2}-U_{s}^{2}\right)^{1 / 2}}{q_{0}^{2}-U_{s} u_{p}}, \\
\sin \beta=\frac{U_{s}}{q_{0}}
\end{gathered}
$$

where the shock is viewed in the Lagrangian frame, as shown in Fig. 2(a). For a wide range of materials, the shock velocity, $U_{s}$, is commonly written as the linear function of the particle velocity, ${ }^{12} u_{p}$, shown in Eq. (3),

$$
U_{s}=c_{0}+s u_{p}
$$

Using the shock conservation equations, ${ }^{1}$ it can be seen that the shock and particle velocities, in a hydrodynamic setting, can be parameterized by the pressure. In this sense, Eqs. (1) and (2) represent the classic P- $\theta-\beta$ shock polar ${ }^{22}$ relationship for an oblique shock in a solid.

Applying the oblique shocks to the Mach lens configuration in the Eulerian frame results in the idealized profile shown in Fig. 3(a). At the interface, the shock in the outer material has an assumed angle, $\xi$, while the incident shock in the inner material is taken to be at an angle, $\eta$. Continuity of the wave front at the interface forces the upstream velocities to be the same. Since the shock velocity in the outer cylinder can be calculated through impedance matching with the impactor, the equation of state for each material can be used in conjunction with Eqs. (1) and (2) to find the unique point at which the mass and momentum are conserved. Graphically, this is shown in Fig. 3(b) as the intersection of the two polar curves in P- $\theta$ space. This will be referred to as the shock polar solution, and completely characterizes the state of reflection at the interface.

\section{NUMERICAL SIMULATIONS}

A series of simulations were conducted on the Mach configuration using the CTH hydrocode. ${ }^{23}$ The 2-D axisymmetric simulations utilized a Mie-Gruneisen equation of state $^{24}$ centered on the typical linear shock-particle velocity Hugoniot governed by Eq. (3), where the empirical coefficients, $c_{0}$ and $s$, for these materials can be found in the literature, $^{12}$ and are listed in Table I. A variety of constitutive

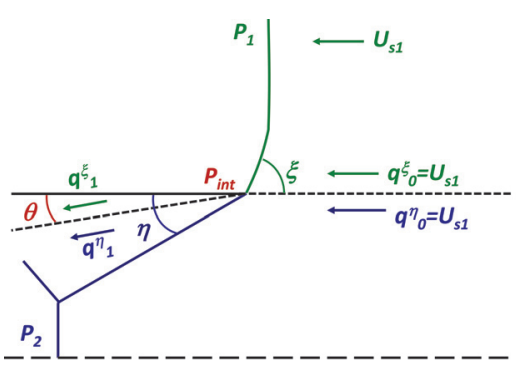

(a)

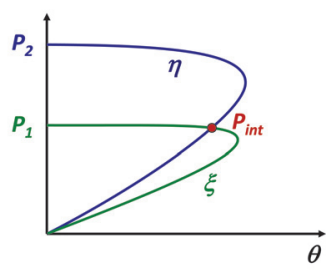

(b)
FIG. 3. (Color online) (a) In the steady state Mach reflection, the shock in the outer material diffracts as it approaches the interface. For simplicity, the Mach reflection in the inner material is assumed to be composed of straight line segments consisting of the incident shock and a normal Mach stem. (b) The shock polar analysis gives an estimate of the state at the interface, including the oblique shock angles and the pressure. The state behind the Mach stem corresponds to the peak of the inner polar.

(strength) models were explored in these simulations, including Johnson-Cook, ${ }^{25}$ Zerilli-Armstrong, ${ }^{26}$ and SteinburgGuinan-Lund. ${ }^{27}$ While none of these models seemed to deviate much from perfect plasticity in the measured shock properties, the subtle effects of strength seem to play a larger role in this configuration than would otherwise be expected in the analogous one dimensional plate impact setup. As such, the built-in Steinburg-Guinan-Lund model was used for the target materials in all of the numerical simulations presented here. The parameters used in the simulations are given in Table II, where the variable definitions can be found in the documented СТH implementation of the model. ${ }^{28}$

Results from a typical simulation showing the development of the Mach reflection are given in Fig. 4. Contour plots at varying times from impact are shown in Figs. 4(a)-4(d) in which the waves are propagating from left to right. The contours are split such that the density is shown in the upper half plane of the plot where the inner and outer materials are evident. The pressure contours shown in the lower half plane give a clear view of the evolution of the Mach reflection. Early in time [Fig. 4(a)], the impedance mismatch results in converging reflected shocks at the interface between the inner and outer materials. Upon convergence [Fig. 4(b)], the 3 wave Mach reflection shown in Fig. 1 forms. The Mach stem grows [Fig. 4(c)] until it reaches a limiting length [Fig. 4(d)] at which point the wave configuration and the properties associated with it no longer change in time. A more quantitative look at this simulation is shown in Fig. 4(e), where equally spaced Lagrangian tracer points along the centerline of the inner cylinder illustrate the evolution of the wave profile. As is shown, the early time particle velocity response is what would be expected in a typical planar

TABLE I. Hugoniot's parameters obtained from least-squares fitting of $U_{s^{-}}$ $u_{p}$ data (Ref. 12) to Eq. (3).

\begin{tabular}{lrcc}
\hline \hline Material & $\rho_{0}(\mathrm{~g} / \mathrm{cc})$ & $C_{0}(\mathrm{~km} / \mathrm{s})$ & $s$ \\
\hline Copper & 8.924 & 3.912 & 1.508 \\
6061-T6 Aluminum & 2.703 & 5.332 & 1.344 \\
Molybdenum & 10.208 & 5.137 & 1.220 \\
Iron & 7.856 & 3.745 & 1.676 \\
\hline \hline
\end{tabular}


TABLE II. Parameters for the Steinburg-Guinan-Lund model (Ref. 28).

\begin{tabular}{|c|c|c|c|c|c|c|c|c|c|c|c|c|c|c|c|c|}
\hline & $\begin{array}{c}\mathrm{Y}_{0} \\
\text { dynes/ } \\
\mathrm{cm}^{2}\end{array}$ & $\begin{array}{c}Y_{\max } \\
\text { dynes/ } \\
\mathrm{cm}^{2}\end{array}$ & $\begin{array}{c}\mathrm{T}_{\mathrm{mo}} \\
\mathrm{eV}\end{array}$ & $\mathrm{a}$ & $\gamma_{0}$ & $\begin{array}{c}\text { A } \\
(\text { dynes/ } \\
\left.\mathrm{cm}^{2}\right)^{-1}\end{array}$ & $\begin{array}{c}\mathrm{B} \\
\mathrm{eV}^{-1}\end{array}$ & $\mathrm{n}$ & $\begin{array}{c}\mathrm{C}_{1} \\
\sec ^{-1}\end{array}$ & $\begin{array}{c}\mathrm{C}_{2} \\
\text { dynes/ } \\
\mathrm{cm}^{2}\end{array}$ & $\begin{array}{c}\mathrm{G}_{0} \\
\text { dynes/ } \\
\mathrm{cm}^{2}\end{array}$ & $\beta$ & $\begin{array}{c}\mathrm{Y}_{\mathrm{p}} \\
\text { dynes/ } \\
\mathrm{cm}^{2}\end{array}$ & $\begin{array}{l}\mathrm{U}_{\mathrm{k}} \\
\mathrm{eV}\end{array}$ & $\begin{array}{c}\mathrm{Y}_{\text {max }}^{0} \\
\text { dynes/ } \\
\mathrm{cm}^{2}\end{array}$ & $\begin{array}{c}\mathrm{Y}_{\mathrm{A}} \\
\text { dynes/ } \\
\mathrm{cm}^{2}\end{array}$ \\
\hline $\mathrm{Al}$ & $2.9 \times 10^{9}$ & $6.8 \times 10^{9}$ & 0.105 & 1.5 & 1.97 & $6.52 \times 10^{-12}$ & 7.15 & 0.1 & 0 & 0 & $2.76 \times 10^{11}$ & 125 & 0 & 0 & 0 & 0 \\
\hline $\mathrm{Cu}$ & $1.2 \times 10^{9}$ & $6.4 \times 10^{9}$ & 0.154 & 1.5 & 2.02 & $2.83 \times 10^{-12}$ & 4.38 & 0.45 & 0 & 0 & $4.77 \times 10^{11}$ & 36 & 0 & 0 & 0 & 0 \\
\hline Mo & $1.6 \times 10^{10}$ & $2.8 \times 10^{10}$ & 0.315 & 1.3 & 1.59 & $1.14 \times 10^{-12}$ & 1.76 & 0.1 & $3.5 \times 10^{7}$ & $1.2 \times 10^{4}$ & $1.25 \times 10^{12}$ & 10 & $1.7 \times 10^{10}$ & 0.372 & $1.6 \times 10^{10}$ & $9 \times 10^{9}$ \\
\hline
\end{tabular}

impact between the flyer and inner material. At $\sim 0.7 \mu \mathrm{s}$ from impact, the converging waves from the interface begin to arrive and the developing Mach reflection significantly increases the particle velocity. At $\sim 3.2 \mu$ s, the reflection has approached the self-similar solution and the wave configuration, which is now analogous to a Taylor wave, becomes steady in time. It should be noted that at the center of the target the Mach stem is confined by the geometry to be normal to the flow direction. Thus, for regions which contain negligible stem curvature, uniaxial strain can be assumed and the conditions are analogous to those in a plate impact experiment. As will be discussed later, however, this does not apply to any precursors to the Mach reflection. In this case, the precursors load the material to a multi-axial state of strain and, so, cannot be correlated to the elasticity observed in plate impact loading.

\section{A. Strong confinement}

The pressure contours for a typical aluminum confinement simulation using the conditions from the MW-O1 experiment (Table III), along with an overlay of the corresponding polar analysis angles, 42.45 and $72.46^{\circ}$ for $\eta$ and $\xi$ [defined in Fig. 3(a)], respectively, are shown in Fig. 5(b). The linear shock-particle velocity relation in Eq. (3) was used to calculate the shock polars using Eqs. (1) and (2), and are shown in Fig. 5(a). As shown, the intersecting shock polars imply that the oblique shocks alone are able to turn the flow to the appropriate state, a case typically referred to as strong confinement ${ }^{29}$ in the detonation community. The Mach disk velocity of $6.18 \mathrm{~km} / \mathrm{s}$ corresponds to a pressure of 83.0 GPa, while стн yields a stress of $81.4 \mathrm{GPa}$ in the direction of propagation. This difference can be attributed to the multidimensional elastic-plastic waves, as seen in Fig. 5(a), preceding the Mach reflection. This nonquiescent state in front of the reflection is a result of material strength, and should be accounted for to accurately calculate the Hugoniot state behind the shock. A simple correction to account for strength will be discussed in the next section. Even with this reduction in stress, the equivalent plate impact experiment, a homogeneous copper target under the same impact conditions will only achieve a stress of $14 \mathrm{GPa}$, which is over a factor of 5 less. Alternatively, to achieve a stress of $81 \mathrm{GPa}$ in this plate impact experiment, an impact velocity of 4.6 $\mathrm{km} / \mathrm{s}$ is required, illustrating the types of gains expected in this experimental configuration.

\section{B. Weak confinement}

The use of a molybdenum outer cylinder results in the weak confinement solution for the MW-02 experiment (Table III) shown in Fig. 6(a). In this case, the shock polars no
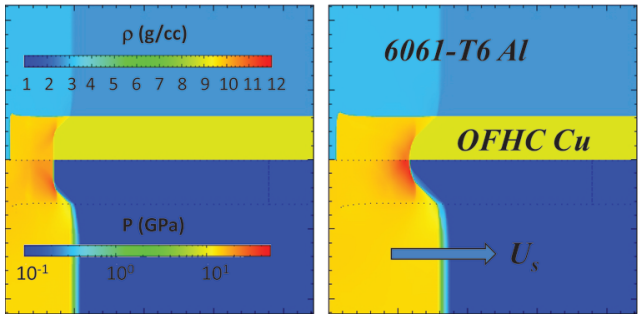

(a) $\mathrm{t}=0.75 \mu \mathrm{s}$

(b) $\mathrm{t}=1.25 \mu \mathrm{s}$

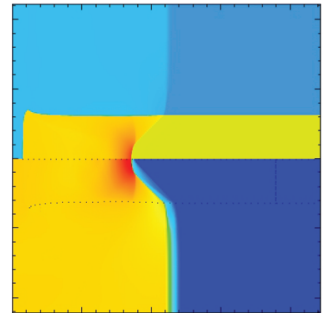

(c) $\mathrm{t}=1.75 \mu \mathrm{s}$

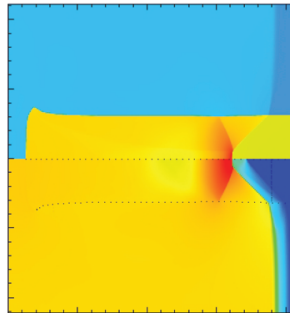

(d) $\mathrm{t}=3.00 \mu \mathrm{s}$

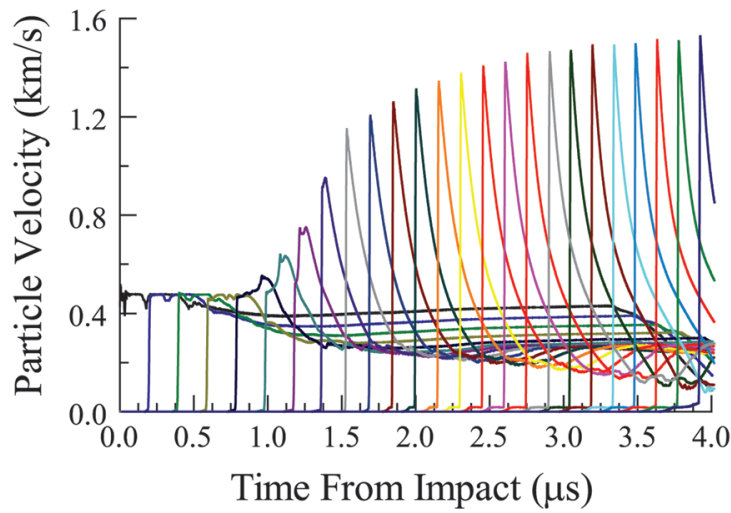

(e)
FIG. 4. (Color online) Simulation of the MW-V1 experiment. (a)-(d) Contours of density in the upper half of the target and pressure in the lower half illustrating the formation and subsequent growth of the Mach reflection are shown. The Lagrangian particle velocity as a function of time at 25 equally spaced points along the length at the center of the target in (e) demonstrates the growth of the wave profile into a steady state. 
TABLE III. Experimental parameters and results.

\begin{tabular}{|c|c|c|c|c|c|c|c|c|}
\hline Exp. No. & Materials $^{\mathrm{a}}$ & $\begin{array}{c}\text { Thickness }^{\mathrm{b}} \\
(\mathrm{mm})\end{array}$ & $\begin{array}{l}\text { Diameter }^{\mathrm{c}} \\
\quad(\mathrm{mm})\end{array}$ & $\begin{array}{l}\mathrm{V}_{\mathrm{I}} \\
(\mathrm{km} / \mathrm{s})\end{array}$ & $\begin{array}{l}U_{S}{ }^{\text {outer }} \\
(\mathrm{km} / \mathrm{s})\end{array}$ & $\begin{array}{l}\mathrm{u}_{\text {peak }} \\
(\mathrm{km} / \mathrm{s})\end{array}$ & $\begin{array}{l}\sigma \\
(\mathrm{GPa})\end{array}$ & $\begin{array}{l}\rho \\
(\mathrm{g} / \mathrm{cc})\end{array}$ \\
\hline MW-V1 & $\mathrm{Al} / \mathrm{Al} / \mathrm{Cu}$ & $12.73 / 22.207$ & $76.71 / 6.414$ & 1.558 & 6.379 & 3.10 & 87.91 & 11.82 \\
\hline MW-V2 & Steel/Al/Cu & $3.08 / 15.824$ & $37.94 / 4.831$ & 1.401 & 6.587 & 3.44 & 100.63 & 12.11 \\
\hline MW-V3 & Steel/Al/Cu & $3.07 / 15.926$ & $37.92 / 4.816$ & 1.310 & 6.506 & 3.28 & 94.32 & 12.01 \\
\hline MW-V4 & $\mathrm{Al} / \mathrm{Al} / \mathrm{Cu}$ & $12.85 / 21.933$ & $76.45 / 6.416$ & 0.787 & 5.861 & \multicolumn{3}{|c|}{ No Mach Reflection } \\
\hline MW-V5 & Steel/Mo/Cu & $3.07 / 15.776$ & $37.85 / 4.805$ & 1.826 & 6.057 & 2.77 & 73.72 & 11.65 \\
\hline MW-V6 & $\mathrm{Al} / \mathrm{Mo} / \mathrm{Cu}$ & $3.16 / 15.766$ & $37.71 / 4.803$ & 1.446 & 5.568 & 1.84 & 44.63 & 10.76 \\
\hline MW-V7 & Steel/Mo/Cu & $3.07 / 15.873$ & $37.85 / 4.829$ & 1.314 & 5.782 & 2.33 & 59.61 & 11.21 \\
\hline MW-V8 & Steel/Mo/Cu & $3.07 / 15.840$ & $37.95 / 4.826$ & 1.043 & 5.641 & 2.062 & 50.99 & 10.98 \\
\hline MW-V9 & $\mathrm{Al} / \mathrm{Mo} / \mathrm{Cu}$ & $3.15 / 15.773$ & $37.93 / 4.821$ & 0.958 & 5.416 & \multicolumn{3}{|c|}{ No Mach Reflection } \\
\hline MW-O1 & $\mathrm{Al} / \mathrm{Al} / \mathrm{Cu}$ & $12.63 / 17.418$ & $50.8 / 4.806$ & 1.241 & 6.166 & $\ldots$ & $\cdots$ & $\ldots$ \\
\hline MW-O2 & $\mathrm{Al} / \mathrm{Mo} / \mathrm{Cu}$ & $12.68 / 18.499$ & $76.2 / 6.414$ & 1.270 & 5.511 & $\ldots$ & $\ldots$ & $\ldots$ \\
\hline
\end{tabular}

${ }^{\mathrm{a}}$ Impactor/outer cylinder/inner cylinder.

${ }^{\mathrm{b}}$ Impactor/target.

${ }^{\mathrm{c}}$ Outer cylinder/inner cylinder.

longer intersect, so an expansion wave in addition to the oblique shock in the outer cylinder is required to deflect the flow to equilibrium. The flow must remain supersonic for the expansion to be realistic, so the sonic point is selected as the maximum turning angle for the oblique shock. The wave speeds and analogous form for the Prandtl-Meyer expan$\operatorname{sion}^{30}$ can be constructed using a Mie-Gruneisen EOS. As shown in Fig. 6(b), the expected Mach profile is very different from the previous case. Here, the Mach stem is much more pronounced, and similar to what has been observed in fluids, ${ }^{31}$ there is a noticeable curvature away from the target centerline. The polar angles of $47.2^{\circ}$ and $75.3^{\circ}$ for $\eta$ and $\xi$ are again in excellent agreement with the simulated profile. Since molybdenum has a lower wave speed than aluminum, there is a reduction in the peak Hugoniot state seen at the center of the target, but this still results in an increase in pressure of over three times the corresponding classic plate impact configuration (and an equivalent plate impact velocity of $3.3 \mathrm{~km} / \mathrm{s})$. The Mach stem pressure is estimated to be $52.2 \mathrm{GPa}$, which is in reasonable agreement with the corresponding numerical result of $50.43 \mathrm{GPa}$.

\section{STRENGTH EFFECTS}

A solid's ability to support shear stresses is expected to result in the reflection of both longitudinal and shear waves. ${ }^{32}$ This results in elastic-plastic precursors to the Mach reflection, and is captured clearly in Figs. 5(a) and 6(a). The stresses generated behind the Mach stem are generally high enough to create shock speeds higher than the longitudinal wave speed, so typical plane wave Hugoniot measurements are made where the shock is jumping from a quiescent state. In this configuration, then, it becomes important to account for the moving state ahead of the shock if any comparisons to previous Hugoniot measurements are to be made. While these reflections are complex and result in a multidimensional state of stress and strain ahead of the shock, it appears to be sufficient to use the measured precursor velocity in the waveform to calculate the correct jump conditions. The Lagrangian jump conditions for a shock propagating into a nonquiescent material are given by ${ }^{33}$

$$
\begin{gathered}
\sigma_{x}=\rho_{0} U_{s}\left(u_{p}-u_{p}^{*}\right)+\sigma_{x}^{*}, \\
\rho=\frac{\rho_{0} \rho^{*} U_{s}}{\rho_{0} U_{s}+\rho^{*}\left(u_{p}^{*}-u_{p}\right)},
\end{gathered}
$$

where $\sigma_{x}$ and $\rho$ are the axial stress and material density, respectively. Here, $\rho_{0}$ is the initial material density, the asterisks indicate the material state ahead of the shock, and the rest of the material properties refer to the shocked state. To

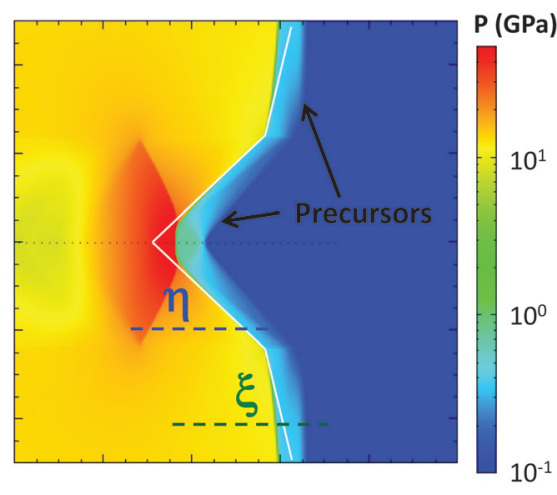

(a)

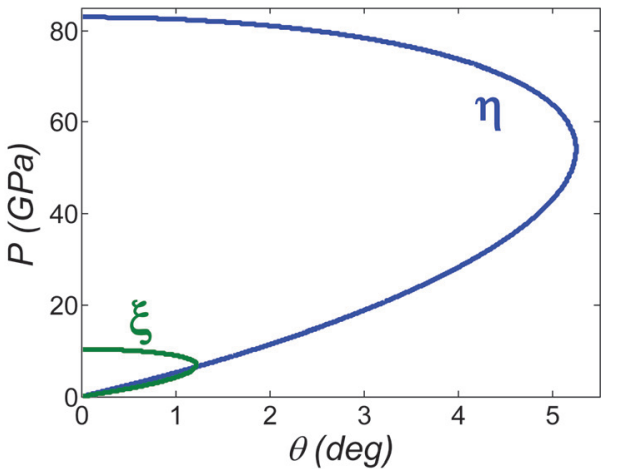

(b)
FIG. 5. (Color online) Simulation of MW-O1 experiment. The shock polar analysis shown in (b) gives the angles overlaid on the simulated pressure contours shown in (a). 


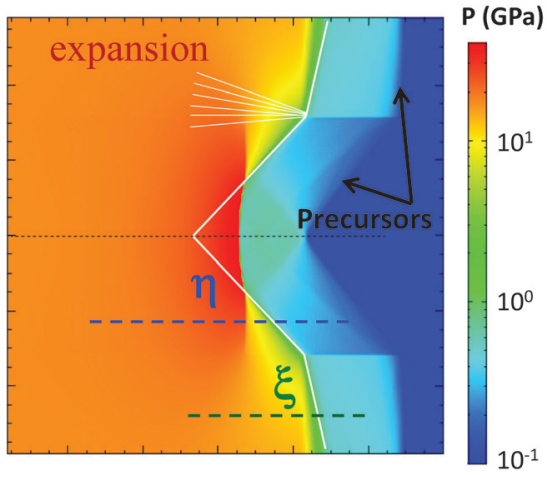

(a)

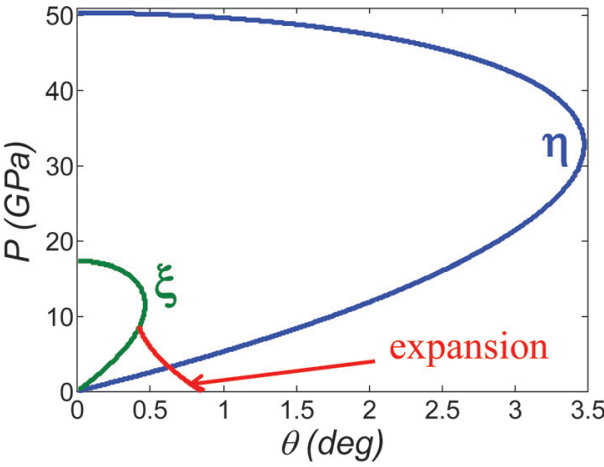

(b)
FIG. 6. (Color online) Simulation of MW-O2 experiment. The shock polar analysis shown in (b) gives the angles overlaid on the simulated pressure contours shown in (a). calculate the state ahead of the shock it is assumed that the deformation is plastic but at a low enough pressure such that the bulk modulus can be taken to be constant. The deformation in the direction of interest $(x)$ then, can be lumped into a single jump which is characterized by the measured precursor velocity, $u_{p}^{*}$, and the bulk wave speed, $c_{0}$. Thus, the stress and density ahead of the shock can be calculated by the quiescent form of the jump conditions,

$$
\begin{aligned}
\sigma_{x}^{*} & =\rho_{0} C_{0} u_{p}^{*}, \\
\rho^{*} & =\frac{\rho_{0} C_{0}}{C_{0}-u_{p}^{*}} .
\end{aligned}
$$

Given the stress and density behind the Mach stem from Eqs. (4) and (5), it is then possible to calculate an equivalent shock and particle velocity in the principle frame by solving the standard mass and momentum Rankine-Hugoniot equations. This analysis is particularly important in the use of the molybdenum confinement where the relative magnitude of the precursor is much higher. This larger precursor effect is a combination of the higher strength of the molybdenum, which results in greater plastic deformation in the copper, and the smaller magnitude of the shock due to the slower wave speed. It should be noted that as the shock in the outer cylinder exceeds the longitudinal wave speed, the precursors in the inner material vanish, making these corrections unnecessary and the material strengths unimportant.

\section{EXPERIMENTAL RESULTS AND DISCUSSION}

\section{A. VISAR experiments}

In an attempt to fully validate the technique, a multipoint VISAR experiment will be discussed in detail. The details of the experimental conditions, MW-V1, are given in Table III and the expected simulated contours were shown previously in Fig. 4. As shown in the inset in Fig. 7(a), the experiment utilized four VISAR probes positioned along the centerline of the target at radii of $0,1.35,2.69$, and $7.21 \mathrm{~mm}$, with dual delay interferometers on the two innermost locations. Along with the resulting waveforms, Fig. 7(a) also contains the $\mathrm{CTH}$ results for tracer locations at the same positions as the experimental probes, along with the results of the shock polar analysis.

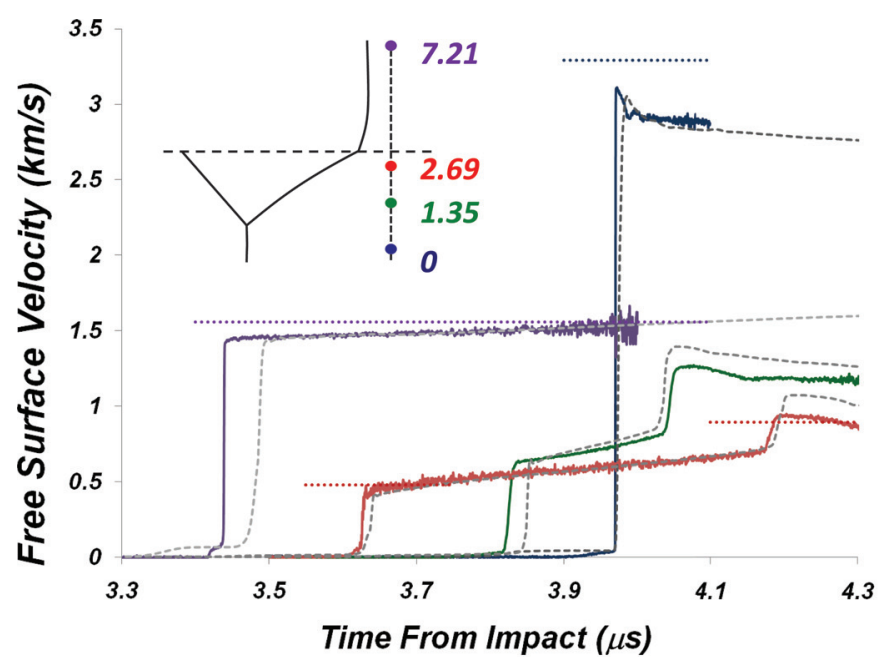

(a)

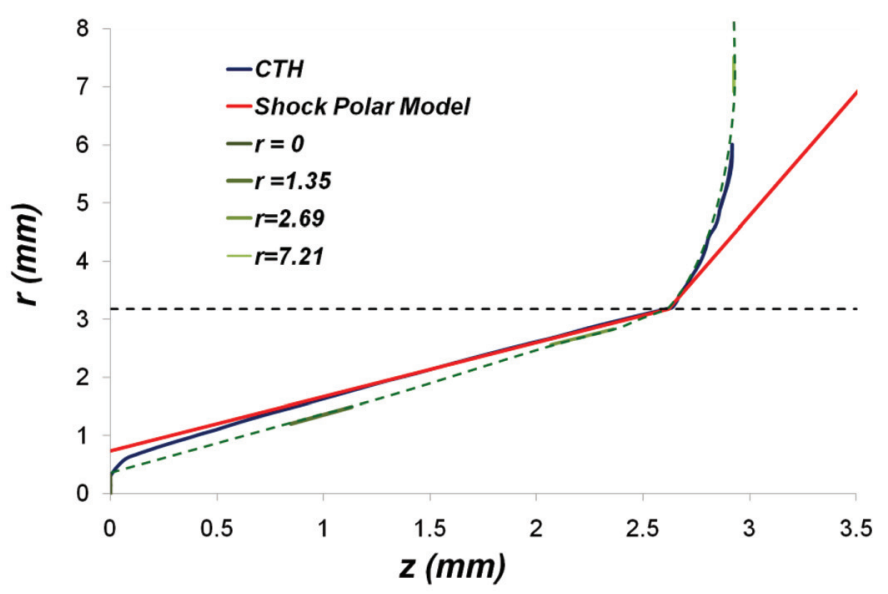

(b)

FIG. 7. (Color online) (a) Waveforms measured in MW-V1 along with results from the numerical simulation (dotted lines) and the shock polar analysis (dashed horizontal lines). The inset shows the position of the four probes along the free surface of the target along with the expected axisymmetric Mach configuration. (b) Calculated Mach wave configuration based on the four measured points as compared to the simulation shown in Fig. 4 and the polar analysis. 
As we expected, at the center of the target, the Mach stem arrives latest in time and shocks the material to a peak state, followed by a release until a loss of contrast occurs in the interferometer. The peak free surface velocity is 3.10 $\mathrm{km} / \mathrm{s}$ (impact velocity, $1.558 \mathrm{~km} / \mathrm{s}$ ), with the simulated value being $1.8 \%$ lower. When the in situ particle velocity calculated from the shock polar analysis is doubled, the resulting velocity is $3.29 \mathrm{~km} / \mathrm{s}$, suggesting the hydrodynamic approximation in this case is good to within approximately $5 \%$.

Increasing in radius, the signal at the probe at $1.35 \mathrm{~mm}$ arrives a couple hundred nanoseconds earlier. Here, a double shock can be seen in the waveform, so it is expected that this probe is monitoring the outer region of the Mach reflection where the incident shock is followed by the reflected shock. The rise in velocity after the initial shock suggests the shock has a degree of curvature which has been shown to produce a pressure gradient in the downstream flow. ${ }^{34}$

The earliest arrival of the Mach reflection occurs at the interface, near the $2.69 \mathrm{~mm}$ radius probe. The waveform looks qualitatively similar to the previous one, except for a few key differences. First, the velocity is lower since the angle of obliquity changes as the wave converges on the appropriate interface pressure. Second, the gradient behind the incident shock is smaller, suggesting less curvature at this point. Finally, the time between the incident and reflected shock is greater. As seen in Fig. 7(a), the angle between these shocks results in a greater separation between the two measurement points, so this is simply a feature of the wave geometry. Also shown in Fig. 6(a) is a line from the polar analysis predicting the reflected shocked state. This was calculated by assuming the flow returns to its original orientation $(\theta=0)$ behind the reflected shock polar. While this simple methodology cannot capture curvature effects, it does seem to predict the magnitude of the jump. Since this measurement is taken close to the interface, another reasonable quantitative comparison can be made, in which case the analytical model and simulation are found to be within $4 \%$ of the experimentally measured value.

The last probe, at a radius of $7.21 \mathrm{~mm}$, is well into the outer cylinder. In this case, the slight effect of the diffraction of the outer shock is seen since the velocity is slightly lower than the expected normal shock particle velocity, which is plotted as the final dashed line in Fig. 6(a). Once again, the ramp after the shock gives some indication as to the degree of curvature of this diffracted wave. It is somewhat surprising to see that this shock arrival time has the largest deviation ( $\sim 40 \mathrm{~ns}$ ) with the simulated arrival, especially since this location should be the easiest to predict. Uncertainty in the shock velocity, however, is estimated to be on the order of $1.5 \%$. Over the relatively long target length, this corresponds to uncertainties in the shock arrival time of $\sim 50 \mathrm{~ns}$, which could account for this deviation.

The analysis can be extended further by taking the experimental peak particle velocities from each probe and relating these to the appropriate pressure on the shock polar, giving the tangent angles shown in Fig. 7(b). As with the shocked states associated with the Mach reflection, both the simulations and the shock polar model are in good agreement with the calculated experimental spatial configuration which is represented by the interpolation between the tangent angles.

The true validation in these experiments, however, is the ability to calculate the shocked state behind the Mach stem. The particle velocity can be estimated by taking half of the free surface velocity, and since the entire configuration is assumed to be steady, the shock velocity can be calculated through impedance matching ${ }^{24}$ as long as the impactor and outer cylinder are well-characterized materials. Once the precursor velocity is measured, Eqs. (4) and (5) may be used to calculate the stress and density. The Hugoniot measurements from these experiments are given in Table III. As shown in Fig. 8, these results are in good agreement with the literature. The calculated uncertainties are typically on the order of 3\% in stress and $0.7 \%$ in density. The uncertainty in shock velocity is determined by the experimental data sets used in impedance matching. A linear regression is performed on the data set to determine the standard error of the empirical coefficients, $c_{0}$ and $s$, and two standard deviations are taken to give a $95 \%$ confidence interval. The uncertainty in the particle velocity is a combination of the experimental errors associated with the VISAR diagnostic and a systematic error accounting for the free surface approximation which is on the order of $2 \%$ for a shock stress of $100 \mathrm{GPa}$.

It should be noted that the lowest pressure shots in each configuration indicate a complete lack of a Mach reflection. While it is possible that the probe was monitoring a twoshock regime rather than the Mach stem due to impactor tilt or probe misalignment, the corresponding simulations also indicate the lack of a Mach wave. Since the effect of material strength at these low velocities is expected to be much more important and there is nothing in the hydrodynamic theory which indicates there should not be a Mach wave, a no-slip solution at the interface which supports the necessary elasticplastic waves could prevent the Mach wave from developing. Of course, these lower stress Hugoniot points can be obtained with traditional plate impact techniques, so the Mach wave experiments inherently lend themselves to high pressure EOS measurements where the hydrodynamic approximation is valid.

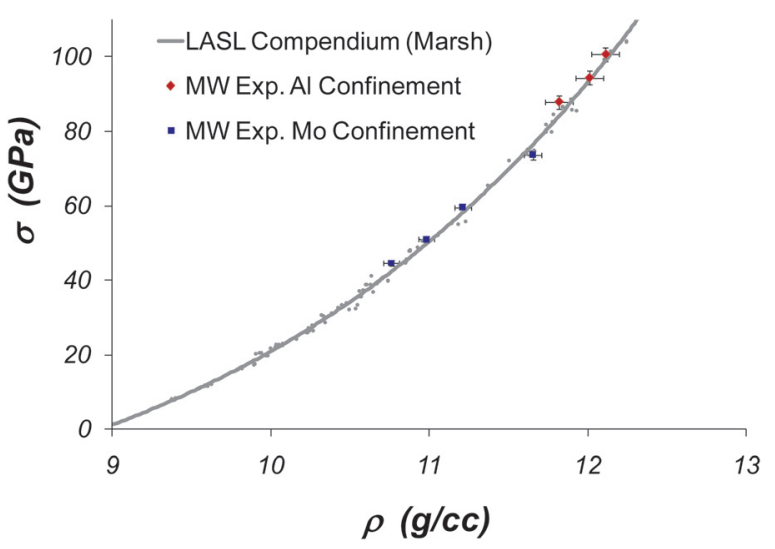

FIG. 8. (Color online) Calculated Hugoniot points for the current experiments along with a comparison to data in the literature (Ref. 12). 


\section{B. ORVIS experiments}

Two experiments were conducted with the ORVIS ${ }^{19}$ diagnostic in an attempt to obtain full field particle velocity information. The first shot, MW-O1 (strong confinement), imaged a line off of a diamond turned rear target surface. The resulting streak camera image, shown in Fig. 9(a), demonstrates many of the features expected in these experiments. The shock arrival at the rear surface creates a clear representation of the initial arrival of the configuration, including the expected elastic precursors, Mach wave, and some evidence of the arrival of the reflected wave. In Fig. 9(b), the precursor and Mach reflection waves from the ORVIS image have been digitized, scaled by the calculated shock velocity, and plotted along with the profiles from Fig. 5. As shown, the simulation seems to do an excellent job of calculating the Mach wave profile and slope of the precursor waves, but the elastic precursor velocity is too high. Unfortunately, only the wave profiles can be calculated, since the loss of light after the initial shock does not allow for the necessary contrast required to extract particle velocity information after the shocks.

In an attempt to remedy the loss of light in the previous experiment, the second shot, MW-O2 (weak confinement), utilized a sandblasted diffuse rear surface. As seen in the ORVIS image in Fig. 10(a), there is much more scatter in the initial fringes, but the overall features expected from this reflection are captured. In this case, not only are the three shocks composing the Mach reflection evident but the Mach stem is much more pronounced and has a measurable curvature. Once again, the digitized image in Fig. 10(b) illustrates the predictive capabilities of both the simulations and the model.

An interesting aspect of this experimental configuration is the inherent pressure gradient in the Mach reflection. Examining a shock polar such as in Figs. 5(b) or 6(b), for example, illustrates the range of pressures involved with the entire reflection. At the center of the target, the symmetry of the problem forces the oblique shock to be normal, so the peak pressure $(\theta=0)$ is achieved. A second known state is the lower bound on the pressure and is achieved at the interface between the two materials, at the point where the two shock polars intersect. Theoretically, then, the rest of the reflection produces continuous states between these two bounds, where the pressure at any point is directly related to the angle of the reflection through the shock polar equations. If, in an experiment, it was possible to measure the angle and peak particle velocity at each point along the reflection, it should be possible to obtain multiple Hugoniot points over a wide range of pressures in a single shot. This was the original intent of the ORVIS experiments, but quality particle velocity information has yet to be collected. As an illustration of the idea, however, a synthetic ORVIS data set was produced from the numerical simulation for MW-V1, and is shown in Fig. 11(a). Given the predictive capabilities of the simulation, illustrated in Fig. 7(a), these traces should be a reasonable approximation to the experiment. A coarse grating, $132 \mu \mathrm{m}$, was used as the spacing between measured points and was chosen as a conservative estimate for an actual ORVIS measurement. From the data set, an instantaneous Mach reflection angle can be approximated between two neighboring velocity traces by examining the shock arrival times. Since the reflection is traveling at a known velocity, $U_{s}$, the instantaneous shock angle, $\beta$, can be approximated by

$$
\beta=\tan ^{-1}\left[\frac{r_{2}-r_{1}}{U_{s}\left(t_{2}-t_{1}\right)}\right],
$$

where $r$ is the radial position of the measured velocity, and $t$ is the arrival time of the shock. The normal components of the shock and average particle velocity, then, are given by

$$
\begin{aligned}
& U_{s}^{\text {normal }}=U_{s} \sin \beta, \\
& u_{p}^{\text {normal }}=\frac{u_{p 1}+u_{p 2}}{4 \sin \beta},
\end{aligned}
$$

where the extra factor of 2 in Eq. (10) accounts for the free surface measurement. Equations (4) and (5), in conjunction with the appropriate component of the precursor velocity, now allow for a calculation of a Hugoniot state for each pair of velocity profiles. Working through this algorithm for the СтH data gives the Hugoniot points shown in Fig. 11(b). Since the CTH simulation is given the copper Hugoniot as an input, the calculated Hugoniot points should, theoretically, lie directly on this curve. The scatter, then, gives us an

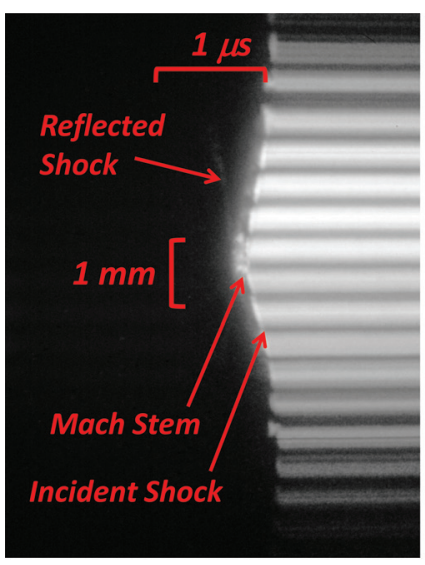

(a)

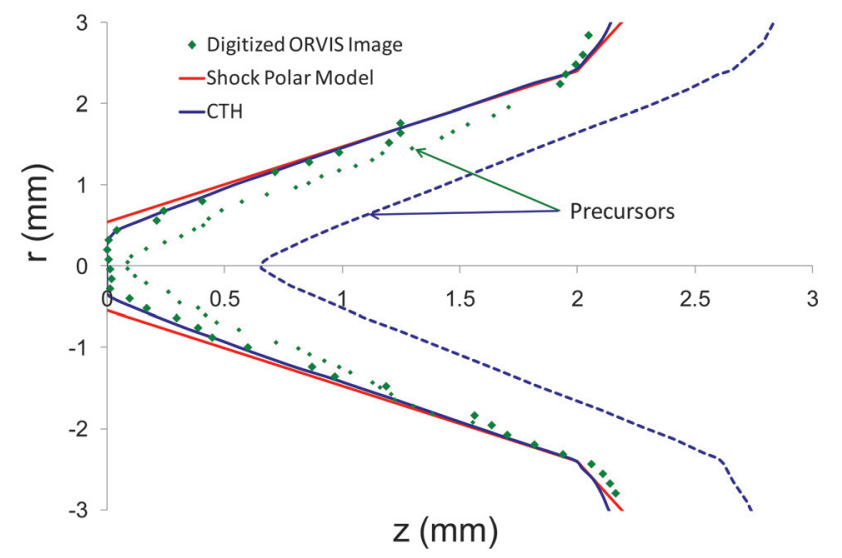

(b)
FIG. 9. (Color online) Experimental results from MW-O1. The ORVIS streak camera image in (a) is digitized to produce the Mach configuration in (b). The experimental results in (b) are overlaid with the simulated and analytical configuration shown in Fig. 5(a). 


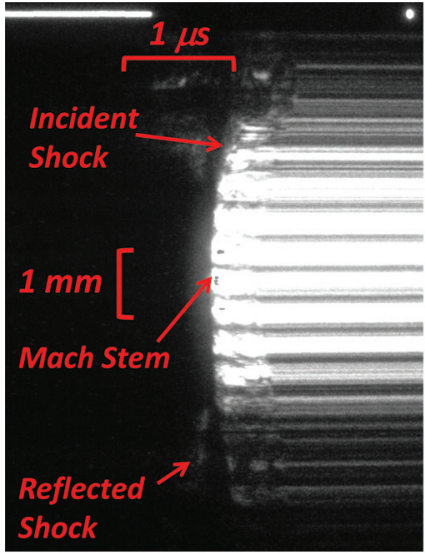

(a)

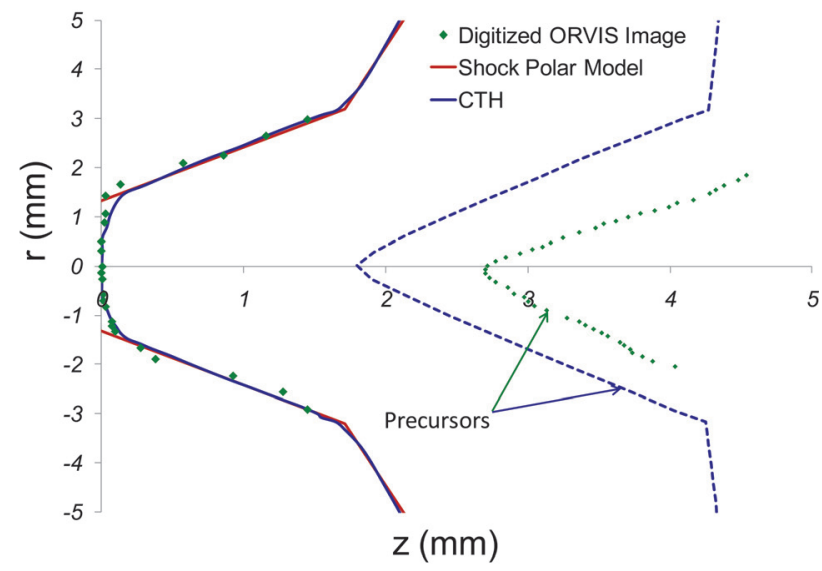

(b)

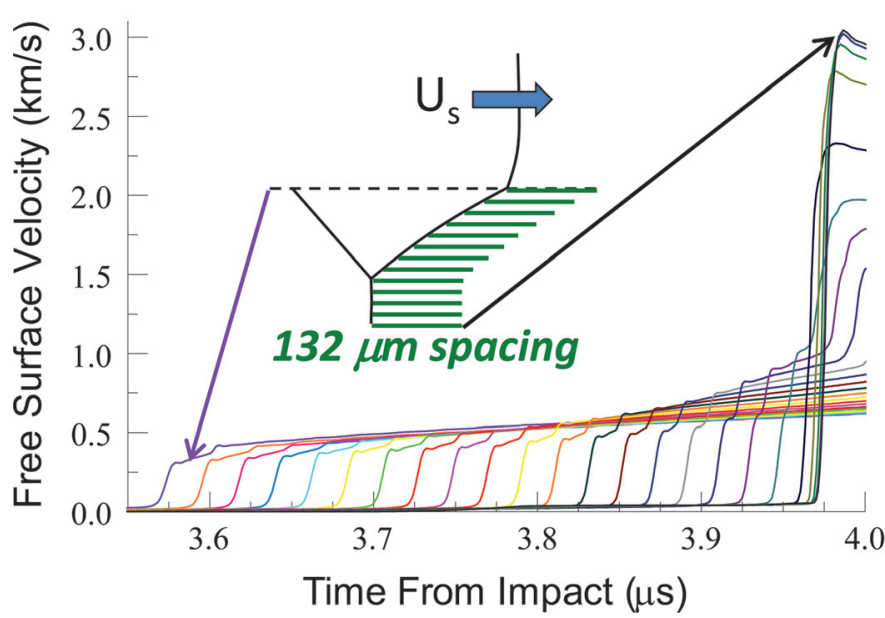

(a)

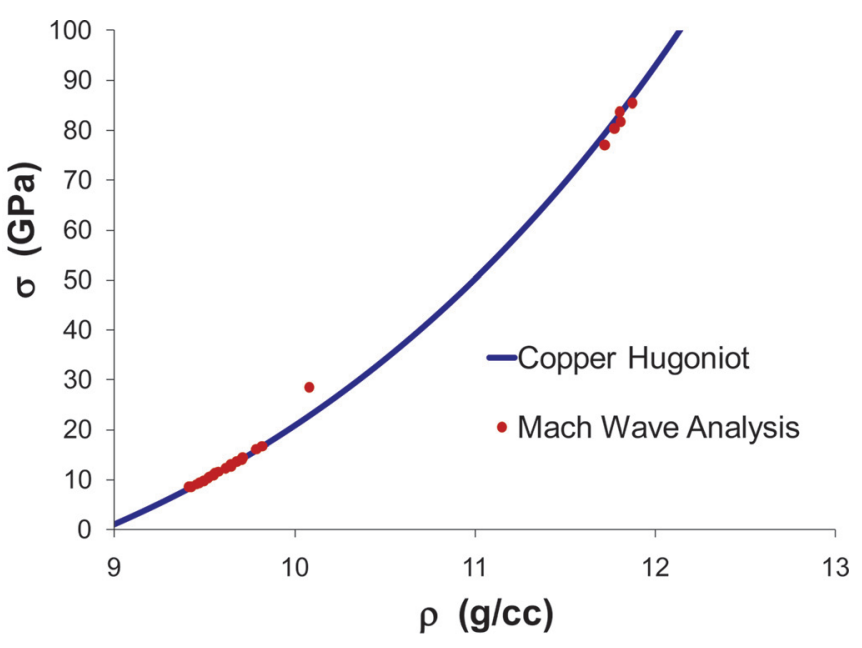

(b)

FIG. 11. (Color online) (a) Synthetic CTH simulation data set for MW-V1, idealizing an ORVIS experiment. Reduction of this data leads to the multiple Hugoniot points seen in (b).

idea of the types of systematic errors associated with choosing arrival times and particle velocities. In particular, there is a stray point associated with the transition between the incident shock and the Mach stem, where the spatial resolution was too low to accurately capture the Hugoniot states between the two. In general, however, given good quality experimental data with high spatial resolution, it seems feasible to calculate a large range of the target material's Hugoniot from a single experiment (shot). Since the form of the Mach reflection can be controlled with the outer material, using a material like molybdenum to produce a more continuous Mach reflection becomes an attractive option for use with this methodology.

\section{CONCLUSIONS}

In the present investigation, the feasibility of using a simple composite target to generate a steady Mach reflection and make high pressure Hugoniot measurements is examined. The steady state velocity of the Mach reflection is determined entirely by the properties of the outer material, so it is possible to calculate the shocked state behind the Mach stem with only a single measurement of the particle velocity. The Mach stem is assumed to be locally planar where this velocity is measured, and when strength effects are taken into account, estimates of the longitudinal stress and density behind the stem are shown to be in good agreement with conventional Hugoniot measurements. The current experiments fall within $3 \%$ of the stress and $1 \%$ of the volume for the copper Hugoniot given in Table I.

With spatial diagnostics such as multipoint VISAR and ORVIS it is possible to characterize the entire Mach reflection. The multipoint methodology provides precision particle velocity information where the Mach stem, incident shock, and reflected shock regimes can be clearly identified through the waveforms. To date, the ORVIS diagnostic has been used to provide the spatial profile of the reflection, but improvements in the current setup are needed to measure the necessary time resolved particle velocity information. 
Several tools have been used to predict and design the experiments. A simple shock polar model has been constructed for solids using hydrodynamic oblique shock theory, where the shocked state behind the Mach stem and at the interface, along with the initial reflection angles can be calculated. A more detailed investigation of the problem has been accomplished using numerical simulations performed with the CTH hydrocode. CTH has been shown to do a reasonable job at predicting not only the waveforms, but also the geometric features of the reflection, particularly the length and curvature of the Mach stem. From a design perspective, the important parameters include the choice of the cylinder materials, cylinder diameters, and the length of the target. For the materials studied here, the simulations suggest that the transition to steady state requires a propagation distance of approximately 2.5 to 3.5 times the inner cylinder diameter. While the outer material dictates how fast the Mach wave will travel, the greater contrast results in longer transition times to steady state and smaller Mach stems. The assemblies are constructed, then, such that the steady state is reached while ensuring the Mach stem is large enough to make a useful measurement. The diameters of the cylinders are chosen such that the release waves from the edge of the outer cylinder cannot affect any of the measurements. Numerical simulations, along with the current experimental results suggest these criteria are met. Verification of the steady state could also be experimentally performed in a convergence study by systematically varying the ratio of the inner cylinder diameter to its length while maintaining constant impact conditions. To date, stresses of up to $100 \mathrm{GPa}$ in copper have been measured using moderate impact velocities (less than $2 \mathrm{~km} / \mathrm{s}$ ). In copper, these stresses can typically only be reached in plate impact experiments through the use of two-stage guns. It is expected that this technique can be used to significantly increase the pressures available to any impact system.

\section{ACKNOWLEDGMENTS}

The research support provided by the Caltech Center for the Predictive Modeling and Simulation of High-Energy Density Dynamic Response of Materials through the U.S. Department of Energy, Contract No. DE-FC52-08NA28613, is gratefully acknowledged. We would also like to acknowledge Tom Thornhill, John Martinez, and Rocky Palomino from Sandia National Laboratories for their technical support in conducting the experiments done at the STAR facility. We thank Professor Hans Hornung for helpful discussions regarding the shock polar analysis, Dr. Dennis Grady for helpful discussions, and Dr. Lalit Chhabildas for his suggestions on shaping the Mach wave.

${ }^{1}$ R. G. McQueen, S. P. Marsh, J. W. Taylor, J. N. Fritz, and W. J. Carter, in High Velocity Impact Phenomena, edited by R. Kinslow (Academic, New York,1970).

${ }^{2}$ W. D. Crozier and W. Hume, J. Appl. Phys. 28, 892 (1957).

${ }^{3}$ L. C. Chhabildas, J. E. Dunn, W. D. Reinhart, and J. M. Miller, Int. J. Impact Eng. 14, 121 (1993).

${ }^{4}$ C. A. Hall, M. D. Knudsen, J. R. Asay, R. Lemke, and B. Oliver, Int. J. Impact Eng. 26, 275 (2000).

${ }^{5}$ M. D. Knudsen, D. L. Hanson, J. E. Bailey, C. A. Hall, and C. Deeney, Phys. Rev. B 69, 144209 (2004).

${ }^{6}$ W. Bleakney and A. H. Taub, Rev. Mod. Phys. 21, 584 (1949).

${ }^{7}$ L. V. Al'tshuler, S. B. Kormer, A. A. Bakanov, A. P. Petrunin, A. I. Funtikov, and A. A. Gubkin, Sov. Phys. JETP 14, 986 (1962).

${ }^{8}$ G. R. Fowles and W. M. Isbell, J. Appl. Phys. 36, 1377 (1964).

${ }^{9}$ G. A. Adadurov, A. N. Dremin, and G. I. Kanel, Zhurnal Prikladnoi Mekhaniki i Teknicheskoi Fiziki 10, 126 (1969).

${ }^{10}$ Y. Syono, T. Goto, and T. Sato, J. Appl. Phys. 53, 7131 (1982).

${ }^{11}$ Y. Mori and K. Nagayama, SPIE High-Speed Photogr. Photon. 1801, 357 (1992).

${ }^{12}$ S. P. Marsh, LASL Shock Hugoniot Data (University of California, Berkeley, 1980).

${ }^{13}$ A. C. Mitchell and W. J. Nellis, J. Appl. Phys. 52, 3363 (1981).

${ }^{14}$ W. J. Nellis, A. C. Mitchell, and D. A. Young, J. Appl. Phys. 93, 304 (2003).

${ }^{15}$ L. C. Chhabildas and J. R. Asay, J. Appl. Phys. 50, 2749 (1979).

${ }^{16}$ D. B. Hayes, R. S. Hixson, and R. G. McQueen, in Shock Waves in Condensed Matter, edited by M. D. Furnish, L. C. Chhabildas, and R. S. Hixson (Elsevier Science, New York, 1999), p. 483.

${ }^{17}$ C. H. Konrad and R. L. Moody, Sandia Report No. SAND86-0791 (1986).

${ }^{18}$ L. M. Barker and R. E. Hollenbach, J. Appl. Phys. 43, 4669 (1972).

${ }^{19}$ D. D. Bloomquist and S. A. Sheffield, Sandia Report No. SAND82-2918 (1982).

${ }^{20}$ D. H. Dolan, Sandia Report No. SAND2006-1950 (2006).

${ }^{21}$ R. Courant and K. O. Friedrichs, Supersonic Flow and Shock Waves (Springer-Verlag, New York, 1948).

${ }^{22}$ H. Hornung, Ann. Rev. Fluid Mech. 18, 33 (1986).

${ }^{23}$ E. S. Hertel, R. L. Bell, M. G. Elrick, A. V. Farnsworth, G. I. Kerley, J. M. Mcglaun, S. V. Petney, S. A. Silling, P. A. Taylor, and L. Yarrington, in CTH: A Software Family for Multi-Dimensional Shock Physics Analysis (Springer, Marseille, France, 1993), pp. 377-382.

${ }^{24}$ M. H. Rice, R. G. McQueen, and J. M. Walsh, in Solid State Physics, edited by F. Seitz and D. Turnbull (Academic Press, New York 1958), Vol. 6.

${ }^{25}$ G. R. Johnson, ASME J. Eng. Mater. and Technol. 103, 201 (1981).

${ }^{26}$ F. J. Zerilli and R. W. Armstrong, J. Appl. Phys. 61, 1816 (1987).

${ }^{27}$ D. J. Steinburg and C. M. Lund, J. Appl. Phys. 65, 1528 (1989).

${ }^{28}$ P. A. Taylor, "CTH Reference Manual: The Steinberg-Guinan-Lund Viscoplastic Model," Report No. SAND92-0716 (1992).

${ }^{29}$ J. W. Banks, W. D. Henshaw, D. W. Schwendeman, and A. K. Kapila, Combust. Theory and Modell. 12, 769 (2008).

${ }^{30} \mathrm{H}$. W. Liepmann and A. Roshko, Elements of Gasdynamics (Dover, New York,1957).

${ }^{31}$ G. Ben-Dor, Shock Wave Reflection Phenomena (Springer-Verlag, New York, 1992).

${ }^{32}$ J. D. Achenbach, Wave Propagation in Elastic Solids (American Elsevier, New York, 1975).

${ }^{33}$ L. Davison, Fundamentals of Shock Wave Propagation in Solids (Springer-Verlag, Berlin, 2008).

${ }^{34}$ H. Hornung, AIAA J. 48, 287 (2010). 